\title{
Alleviating the Adverse Effects of Residual Stress in RF MEMS Switches
}

\author{
Dimitrios Peroulis, Sergio P. Pacheco, Kamal Sarabandi, and Linda P. B. Katehi, \\ Radiation Laboratory, Electrical Engineering and Computer Science Department, \\ University of Michigan, 1301 Beal Ave., Ann Arbor, MI 48109-2122, \\ dperouli@engin.umich.edu, katehi@engin.umich.edu.
}

\begin{abstract}
This paper presents two methods for counteracting the unwanted deflection due to warping or buckling effects, which are serious potential problems in many fabrication processes of microelectromechanical (MEMS) structures due to thin film phenomena. This study is primarily suited for electrostatically actuated RF MEMS switches whose RF and DC performance can be significantly deteriorated by out of plane warping. It can also be applied to MEMS accelerometers, resonators and other similar systems. The first technique focuses on modifying the support structure and the spring constant of the switch, while the second involves a more complicated fabrication process, which selectively increases the switch thickness. Both these techniques yield switches with two to ten times less warping under the same fabrication conditions. The second method, however, presents the additional advantage of maintaining the actuation voltage almost unaffected.
\end{abstract}

\section{INTRODUCTION}

$\mathrm{W}$ ITH ever increasing demand for low power wireless systems, the need for microelectromechanical (MEMS) components is on the rise. Due to their extremely low power consumption and negligible intermodulation distortion, RF MEMS are emerging as a promising alternative to solidstate devices. In particular, MEMS switches with very low insertion loss and high isolation have already been reported [1], [2], [3], [4] and have been successfully integrated with reconfigurable antennas [8], filters [5] and phase shifters [6], [7].

Nevertheless, the performance of these devices can significantly be compromised by fabrication issues such as the adverse effects of residual stresses. Warping and buckling are among the most severe and frequently occurring problems. Furthermore, RF MEMS switches usually face more severe stress problems because of the extensive use of high intrinsic stress metals. Additionally, requirements for reasonably low actuation voltages frequently impose specific limitations on the switch thickness, thus making flat structures more difficult to obtain.

These issues are usually treated by ameliorating the available technology and improving the understanding of the stress issues involved in the specific fabrication processes. In addition, there are also relatively simple design techniques that can significantly contribute to this effort. It is the purpose of this paper to present two of these methods for a previously developed RF switch [1]. The first method considerably mitigates the effect of residual gradient stress by slightly modifying the springs of the switch, while the second one maintains almost the same spring constant and results in essentially flat structures by selectively increasing the switch thickness.

\section{First technique : Meander effects on WARPING}

Fig. 1a shows a low actuation voltage switch whose performance was reported in [1]. This switch is fabricated over a coplanar waveguide (cpw) line and has three movable plates, one over each conductor of the cpw. The four meanders shown on the figure, which have been specifically designed for low actuation voltage, connect the switch to each of the four anchor points . Each of the meanders can be accurately modeled as a serpentine guidedend cantilever beam [9] and the spring constant of the total structure is four times that of a single meander.

The previous simple analysis can approximate the switch actuation voltage according to the formula [1]:

$$
V_{p}=\sqrt{\frac{8 K_{z} g_{0}^{3}}{27 A \epsilon_{0}}}
$$

where $K_{z}$ is the spring constant in the z-direction, $g_{0}$ is the initial gap between the switch and the bottom electrode, $A$ is the area of the actuation pads and $\epsilon_{0}$ is the free space permittivity. Although this formulation is useful for spring constant and actuation voltage calculations, it ignores 

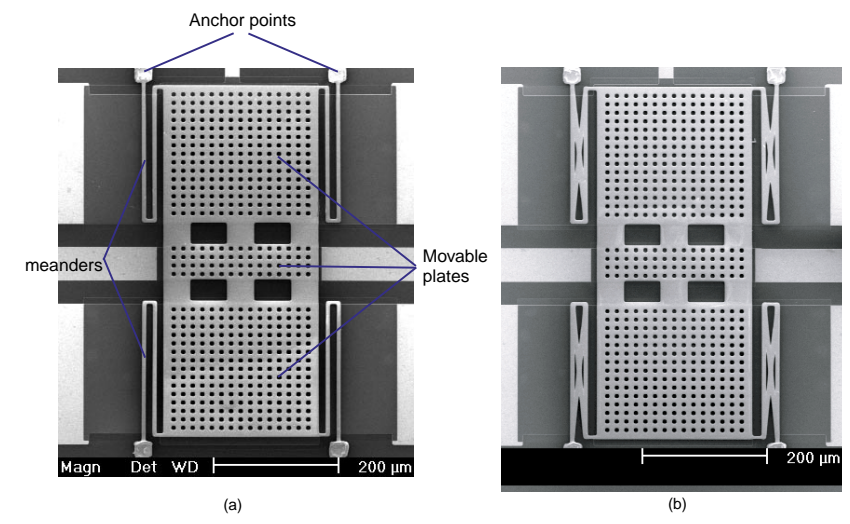

Fig. 1. (a) Low actuation voltage MEMS switch. (b) Same switch structure with different meander sections

all non-linear effects as well as biaxial and gradient stresses, which may dominate the switch performance by making the switch much stiffer, or by introducing significant warping. Formula (1) also implies that $V_{p}$ is more sensitive to $g_{0}$ rather than $K_{z}$ variations. However, the switch deflection due to residual stress may not be independent of its spring constant. In other words, by increasing the spring constant of the switch a smaller deflection can be achieved resulting in a switch with lower actuation voltage than the original one.

For instance, the spring constant for the switch at hand is dominated by the four meanders shown in Fig. 1. The effect of these meanders in the warping of the total structure was theoretically and experimentally investigated by designing, fabricating and measuring switches with eleven different meanders shown in Fig. 2.

The complete fabrication process of the switch is described in [1]. However, the steps that primarily determine the stress distribution on the switch is the seed layer deposition and the nickel electroplating of the moving structure. A combination of these that resulted in significant residual stress were utilized in this study. The same seed layer (evaporated $\mathrm{Ti} / \mathrm{Ni}$ 1500/500 $\AA$ ) and $\mathrm{Ni}$ solution maintained at constant temperature and $\mathrm{pH}$ levels were employed for all the switches. In addition, all switches were electroplated at $5 \mathrm{~mA} / \mathrm{cm}^{2}$ for a total thickness of $2.5 \mu \mathrm{m}$. Fig. 1 shows two of these switches with meanders (a) and (g).

The warping level - defined as the maximum vertical distance between any two points of the moving structure - was measured for each switch with an optical microscope. Fig. 3 summarizes the obtained results by averaging over many switches for
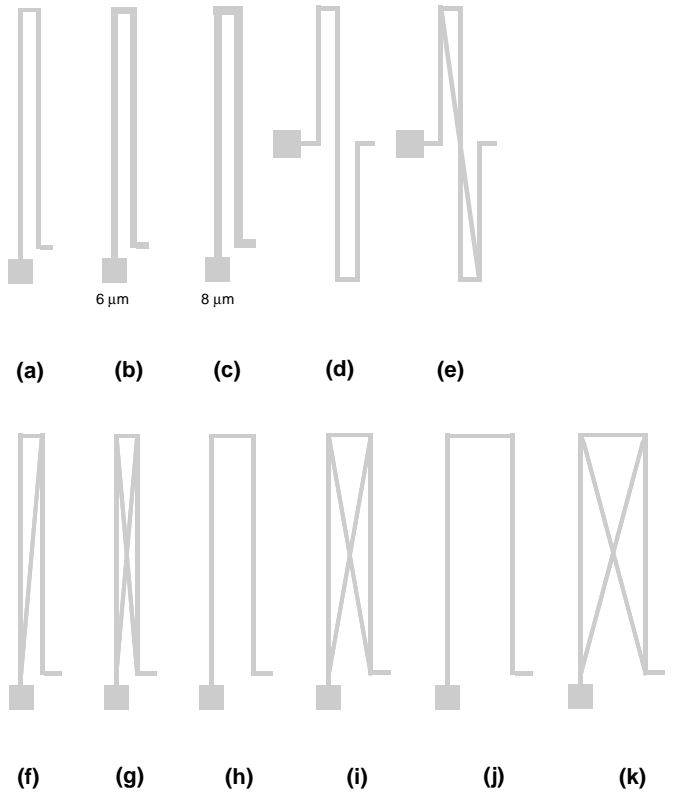

Fig. 2. Eleven different meander designs. Each arm width is $4 \mu \mathrm{m}$, except for (b) and (c) which is 6 and $8 \mu \mathrm{m}$ respectively. Each meander is $240 \mu \mathrm{m}$ long and they are drawn to scale.

each meander type. Deflection measurements are accomplished with an accuracy of the order of 1 $\mu \mathrm{m}$. This figure clearly illustrates that there exist significant variations in the deflection level between the different spring types. For instance, the minimum measured deflection was $4 \mu \mathrm{m}$ and was obtained with spring (g), while spring (j) resulted in the maximum deflection of $27 \mu \mathrm{m}$.

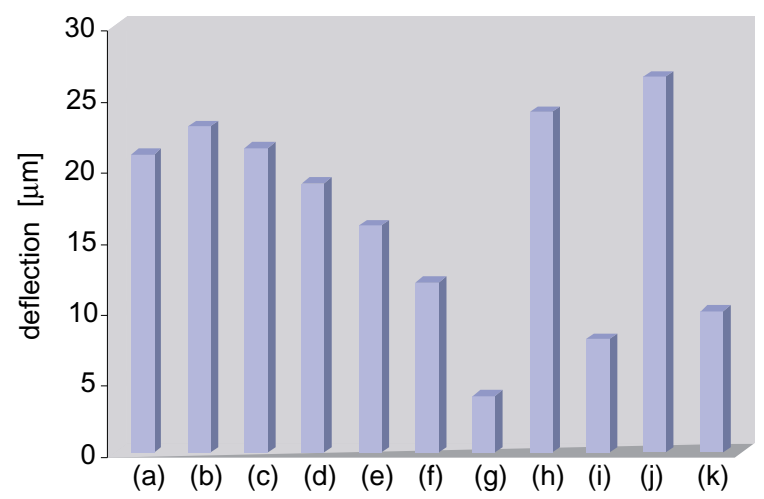

Fig. 3. Average measured deflection for switches using different meanders.

The switch shape under residual gradient stress was also theoretically validated using SUGAR [10], a system-level analysis tool for MEMS simulation and design developed by the Berkeley Sensors and Actuators Center. Fig. 4 shows this switch shape under a residual stress of $150 \mathrm{MPa} / \mu \mathrm{m}$. This picture accurately illustrates the switch shape under this specific stress. 


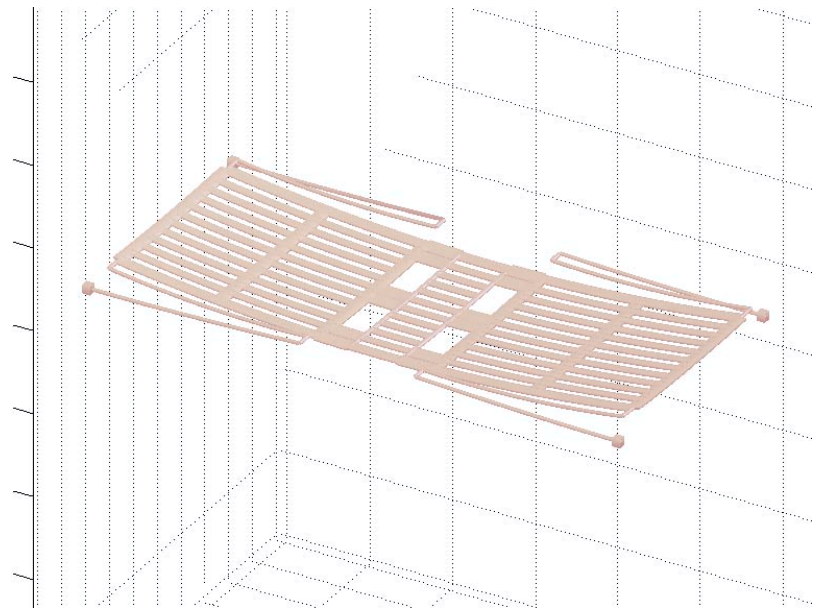

Fig. 4. Simulated shape of a $2.5 \mu \mathrm{m}$ switch under severe positive gradient stress. The total length and width of the switch are 650 and $240 \mu \mathrm{m}$ respectively. The maximum deflection is $23 \mu \mathrm{m}$.

Although significant improvements can be observed to the deflection level by using different meander sections, it is clear that their spring constants varies. Intuitively the stiffer meanders result in less warping, but also in higher actuation voltage, if ideal conditions were to be considered. Therefore, a compromise is necessary and the solution can be found if both these factors are considered. Table I summarizes the spring constants for the meanders considered in this investigation. These values were computed by a commercially available finite element analysis (FEA) software [11]. For simplicity reasons, each meander was simulated with the same boundary conditions as a simple cantilever rather than a guided-end cantilever, which is closer to the actual structure. Nevertheless, since the goal is to compare their spring constants, this simplification is an acceptable approximation. However, the spring constant of the actual meander will be approximately four times higher than the one reported in Table I [9]. Finally, the width of the meanders was increased by $1 \mu \mathrm{m}$ in the simulations to accommodate for the width increase during the plating process. The information conveyed from both Table I and Fig. 3 should be considered in determining the meander type to be used for minimum warping and spring constant. For example, meander (g) is ten times stiffer than (a) and, as a result, the actuation voltage would be increased by a factor of 3.3. However, the warping level for the switches with meander $(\mathrm{g})$ is six times lower than those with meander (a) and thus they eventually have lower actuation voltage. Indeed, the measured voltages for switches with
TABLE I

Calculated SPRing CONSTANTS OF the Meanders in Fig. 2. All the meanders have BeEn SIMULATED AS FREE-END CANTILEVERS

\begin{tabular}{|c|c|c|c|}
\hline meander & $K_{z}(\mathrm{~N} / \mathrm{m})$ & meander & $K_{z}(\mathrm{~N} / \mathrm{m})$ \\
\hline (a) & 0.065 & $(\mathrm{~g})$ & 0.706 \\
\hline$(\mathrm{b})$ & 0.086 & $(\mathrm{~h})$ & 0.057 \\
\hline (c) & 0.105 & $(\mathrm{i})$ & 0.625 \\
\hline (d) & 0.185 & $(\mathrm{j})$ & 0.050 \\
\hline (e) & 0.271 & $(\mathrm{k})$ & 0.538 \\
\hline (f) & 0.094 & & \\
\hline
\end{tabular}

meanders (a) and (g) were approximately 75 and $40 \mathrm{~V}$ respectively. On the other hand, although meanders (d) and (e) are 2.8 and 4.2 times stiffer than (a), they do not contribute a significant improvement to the total warping level of the switch (see Fig. 3) and they should not be used with this type of switch. The actuation voltages for these switches were measured higher than $70 \mathrm{~V}$.

\section{Second Technique : Selective EleCtroplating}

The results of the previous section indicate that the meander design is one of the factors that determine the unwanted deflection of the switch due to residual stress. The other major factor is the switch thickness. In other words, thick switches show far less unwanted deflection than thinner ones. Nonetheless, the spring constant of a cantilever beam is proportional to the third power of its thickness, t, [9] and thus the actuation voltage is proportional to $t^{3 / 2}$.

Although the actuation voltage depends on the meander thickness, it is theoretically independent of the actuation pads thickness. Consequently, the thickness of the three plates shown in Fig. 1 can be increased while keeping the meander thickness at a minimum.

For the switch design at hand, this can be accomplished by selectively electroplating parts of the switch. The plating process is as follows. After the anchor points are defined, $2000 \AA$ of Ti are sputtered followed by $500 \AA$ evaporated Ni. This seed layer is patterned and plated to $2 \mu \mathrm{m}$ in a $\mathrm{Ni}$ solution at $50 \mathrm{C}$ for about $30 \mathrm{~min}$. This first plating defines both the four meanders and the three movable plates of the switch. After thoroughly removing the photoresist, another lithography is performed. This new photoresist is etched only on top 
of the movable plates and not on the meanders. Then the sample is electroplated again under the same conditions for another $4-5 \mu \mathrm{m}$. As a result, the main switch structure is 6-7 $\mu \mathrm{m}$ thick, while the meander thickness remains constant to $2 \mu \mathrm{m}$. The seed layer is then etched and the sacrificial layer is removed in hot PRS2000. Finally, the sample is dried in a supercritical $\mathrm{CO}_{2}$ process.

Although the process was in general successful, after many iterations it was found that the adhesion between the two plated Ni layers was poor and should be improved. This adhesion problem was addressed by plating only a frame of the switch during the first plating. Then the second layer of plated Ni sticks to both the seed layer and the first plating. This technique considerably improved the adhesion between the two Ni layers and resulted in an extremely high yield (higher than 98\%).

This process was experimentally validated by fabricating switches with $2 \mu \mathrm{m}$ thick meanders and $6 \mu \mathrm{m}$ plate thickness. A typical switch is shown in Fig. 5. The measured warping level was 1-2 $\mu \mathrm{m}$ independently of the meander type used and a typical actuation voltage is shown in Fig. 6 .

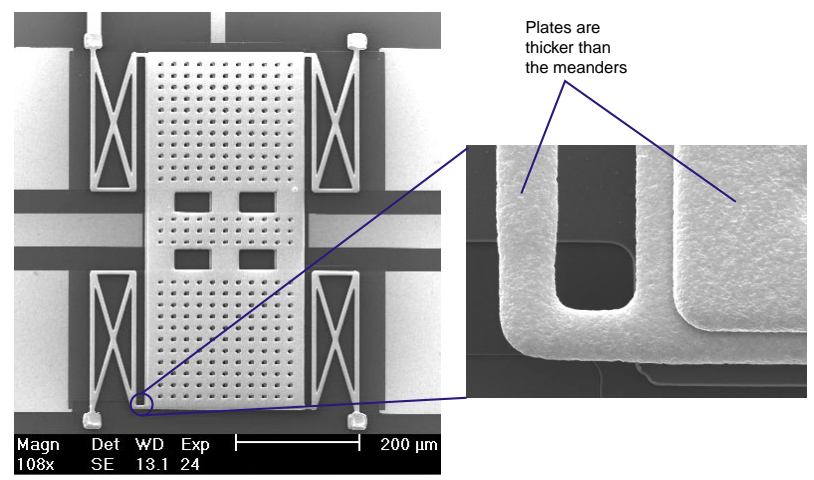

Fig. 5. Switch with thick plates and thin meander sections.

\section{Conclusion}

Two methods for alleviating the unwanted deflection caused by residual stress in RF MEMS switches have been reported. The first technique yields switches with up to six times less warping by compromising the switch spring constant. The second one improves the switch warping up to ten times by selectively thickening these parts of the switch that do not affect its actuation voltage. Although the proposed methods have been demonstrated for a specific switch and fabrication process, they can readily be applied to many others.

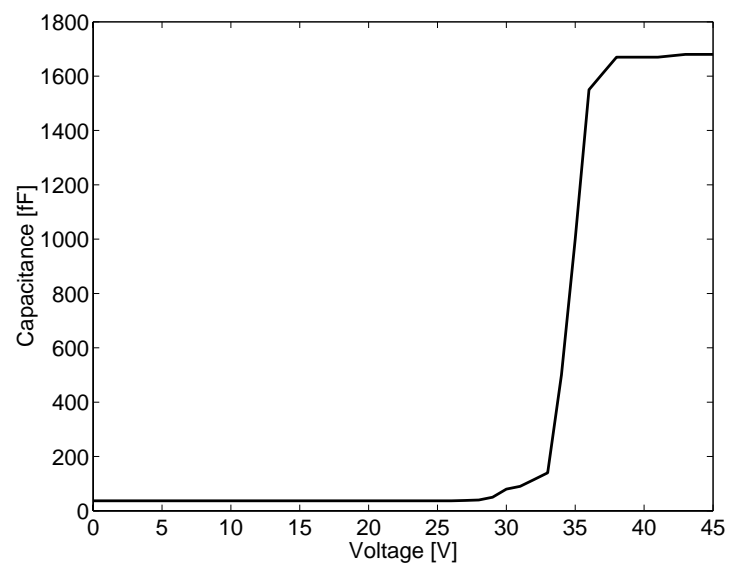

Fig. 6. Switch capacitance as a function of pull-in voltage.

\section{ACKNOWLEDGMENTS}

This work has been supported by DARPA under the RECAP project.

\section{REFERENCES}

[1] S. Pacheco, L. P. B. Katehi, C. T. Nguyen, Design of Low Actuation Voltage RF MEMS Switch, ,IEEE International Microwave Symposium Digest, Vol.1, June 2000, pp. 165-168.

[2] D. Peroulis, S. Pacheco, K. Sarabandi, L.P.B. Katehi, MEMS devices for High Isolation Switching and Tunable Filtering, IEEE International Microwave Symposium Digest, Vol.2, June 2000, pp. 1217-1220.

[3] J. B. Muldavin, G. M. Rebeiz, High-Isolation CPW MEMS Shunt Switches - Part 2: Design, IEEE Transactions on Microwave Theory and Techniques, Vol. 48, no. 6, June 2000, pp. 1053-1056.

[4] C. Goldsmith, J. Randall, S. Eshelman, T. H. Lin, D. Denniston, S. Chen, B. Norvell, Characteristics of Micromachined Switches at Microwave Frequencies, IEEE International Microwave Symposium Digest, Vol.2, June 1996, pp. 1141-1144.

[5] D. Peroulis, S. Pacheco, K. Sarabandi, L.P.B. Katehi, Tunable Lumped Components with Applications to Reconfigurable Filters, Submitted to IEEE International Microwave Symposium Digest,May 2001.

[6] N. S. Barker, Distributed MEMS Transmission Lines, Ph.D. Dissertation, University of Michigan, 1999.

[7] J. S. Hayden, G. M. Rebeiz, One and Two-Bit Low-Loss Cascadable MEMS Distributed X-Band Phase Shifters, IEEE International Microwave Symposium Digest, Vol.2, June 2000, pp. 161-164.

[8] J. C. Chiao, Y. Fu, J. M. Chio, M. DeLisio, L. Y. Lin, MEMS Reconfigurable Antenna, IEEE International Microwave Symposium Digest, Vol.2, June 1999, pp. 1515-1518.

[9] Gary Keith Fedder Simulation of Micromechanical Systems, Ph.D Dissertation, University of California at Berkeley, 1994.

[10] http://bsac.eecs.berkeley.edu/ cfm/.

[11] CADRE 1.3 finite element structural analysis software by CADRE Analytic. 